\title{
Téoros
}

Revue de recherche en tourisme

\section{Pour plus d'harmonie entre la faisabilité et le financement}

\section{Gilles Delorme}

Volume 5, numéro 2, juillet 1986

Tourisme, fêtes et événements

URI : https://id.erudit.org/iderudit/1080575ar

DOI : https://doi.org/10.7202/1080575ar

Aller au sommaire du numéro

Éditeur(s)

Université du Québec à Montréal

ISSN

0712-8657 (imprimé)

1923-2705 (numérique)

Découvrir la revue

Citer cet article

Delorme, G. (1986). Pour plus d'harmonie entre la faisabilité et le financement. Téoros, 5(2), 24-40. https://doi.org/10.7202/1080575ar d'utilisation que vous pouvez consulter en ligne.

https://apropos.erudit.org/fr/usagers/politique-dutilisation/ 


\title{
Pour plus d'harmonie entre la faisabilité et le financement
}

\author{
par Gilles Delorme*
}

\begin{abstract}
Chaque année, les gouvernements municipaux, provinciaux et fédéral ainsi que les entreprises privées sont sollicités pour s'associer à des projets à vocation culturelle ou sportive et, ainsi, bénéficier des retombées positives engendrées par une augmentation des activités économiques aux plans local, régional ou national. À plusieurs occasions, ces associations entre les organisateurs de projets et les promoteurs publics et privés ont permis d'atteindre de façon inespérée les objectifs visés et ainsi de développer un esprit de fierté auprès des populations, de générer une activité commerciale bénéfique et de susciter un intérêt touristique dont toutes les composantes socio-économiques ont pu bénéficier. Plusieurs de ces événements sont d'ailleurs réédités sur une base annuelle et contribuent à la notoriété internationale de certaines régions ou villes du Québec. Malheureusement, les résultats espérés n'ont pas toujours été aussi heureux. Bon nombre de projets ont laissé un souvenir qui ne rend pas justice aux efforts fournis par l'ensemble des travailleurs ayant participé à leur réalisation, et/ou un déficit financier assumé par les contribuables, non proportionnel aux retombées économiques escomptées et de toute façon, beaucoup plus complexes à évaluer.
\end{abstract}

\section{Particularités des projets à vocation culturelle ou sportive} La mise sur pied de projets à caractère sportif ou culturel présente des particularités qui rendent aléatoire le niveau de réussite escompté. L'équilibre à trouver entre la qualité, le coût et le respect de l'échéancier laisse peu de possibilités de manoeuvre aux réalisateurs du projet. Le caractère unique des evénements à réaliser ou l'importance d'innover d'année en année pour en augmenter l'intérêt obligent les gestionnaires à s'adapter et à réagir constamment à des situations nouvelles ou exceptionnelles. Plus que tout autre, ces projets à vocation culturelle ou sportive doivent être publicisés car leur crédibilité

-Gilles Delorme est conseiller en gestion et associé de CERAC Inc., cabinet conseil en administration, planification et évaluation. et leur réussite y sont intimement liées. Et, malgré tout, la différence entre la réussite et l'échec peut dépendre d'éléments n'étant absolument pas contrólables par les organisateurs: la température, les grèves ou autres imprévus.

Parallèlement à ces aspects particuliers, la réalisation d'événements culturels ou sportifs a été influencée par un ensemble de pressions sur les principaux groupes s' $y$ intéressant. Les gouvernements sont incités à réduire leur déficit et à mieux gérer leur budget. On s'attend à une plus grande implication de l'entreprise privée à titre de bon citoyen corporatif. Il est aussi considéré normal que les gestionnaires de ces événements atteignent une performance se comparant à l'efficacité du secteur privé et qu'elle se traduise par un rendement financier plutôt qu'économique. Enfin, les contribuables sont de plus en plus sensibles aux coûts élevés qu'engendre une majorité des projets de cette nature et ils n'acceptent pas nécessairement de les percevoir comme prioritaires ou comme des investissements pouvant amener des retombées économiques et sociales à moyen et long termes.

Ces multiples facettes ont amené les promoteurs des secteurs publics et privés, sollicités pour le financement de ces événements, à se doter d'objectifs bien définis et d'outils d'évaluation qui standardisent le processus d'approbation des projets soumis et protègent leurs intérêts spécifiques. Les concepteurs se trouvent donc dans l'obligation d'adapter leurs requêtes aux règles du jeu établies. Ils doivent développer une stratégie qui tient compte des objectifs et normes d'approbation des éventuels bailleurs de fonds et, de ce fait, adapter la réalité des composantes opérationnelles de leur projet à l'intérieur du cadre régissant ces normes; c'est le seul moyen d'obtenir du financement pour la planification, la réalisation et souvent aussi la survie de l'événement auquel ils croient.

\section{Objectifs et normes d'approbation des promoteurs}

Le financement et l'aide consentis aux projets ne peuvent provenir que des sources suivantes: les subventions gouvernementa- les (qui s'inserrent dans le cadre de programmes bien définis et qui visent des objectifs précis reliés aux orientations des ministères ou organismes qui les régissent), les investissements du secteur privé (qui s'intègrent à leur plan régional ou national de marketing) et le public (qui peut lui aussi participer au financement de certains projets par le biais de la billetterie ou par l'achat de biens et services à des concessionnaires). Cette dernière forme de financement n'est pas toujours applicable - feux d'artifices - et présente le désavantage majeur de n'apporter des fonds qu'à la fin de la planification ou de la campagne de publicite et surtout lors des quelques jours qui précèdent la réalisation de l'événement. Les entreprises privées et publiques seront aussi en mesure d'offrir des services professionnels de qualité ou de $l^{+}$équipement. Toutefois, les organismes gouvernementaux n'étant pas mandatés pour concurrencer les intérêts privés, les services offerts seront réservés à des domaines particuliers tels la santé et la sécurité. L'intervention du secteur privé, pour sa part, sera directement reliée à ses produits et l"utilisation avantageuse qui pourra en être faite nécessitera d'accorder à l'entreprise l'exclusivité. Finalement, un des soutiens majeurs que peuvent accorder les organisations publiques ou privées et surtout les dirigeants de celles-ci, c'est la crédibilité. Le parrainage formel d'un homme politique, d'un ministère ou d'une entreprise de prestige donne beaucoup de poids a l'ampleur du projet et génère une synergie entre les promoteurs éventuels, avec comme principal avantage d'accélérer le processus décisionnel.

Ces réalités ont obligé les gouvernements des niveaux fédéral, provincial et municipal à répondre à plusieurs centaines de requêtes en vue de recevoir de l'aide tech. nique et surtout financière. Les personnes approchées pour répondre à ces requêtes se situent à deux niveaux. Celui des hommes politiques, composé principalement de ministres et de hauts-fonctionnaires. Ces personnes se doivent d'être très prudentes dans leurs engagements; ils ont une connaissance plus limitée des détails relatifs aux programmes qu'ils gèrent et le pouvoir 
légitime d'interpréter ou de modifier les règlements de ces programmes. Les députés et le personnel de cabinet ont pour leur part un pouvoir d'influence important. Les fonctionnaires, second niveau de personnes approchées pour obtenir de l'aide, sont mandatés pour aider à la réalisation d'un projet. Ils doivent cependant le faire à l'intérieur de programmes normés biens définis. Ces personnes ne sont pas autorisès à interpréter ou modifier les règles qui régissent les programmes mais à les appliquer, Leur pouvoir n'en est pas un d'approbation mais de recommandation.

Dans la mesure où les subventions visent la réalisation de projets à caractère sportif ou culturel, les paramètres d'évaluation vont généralement considérer les ou une partie des facteurs suivants: l'importance des retombées économiques et commercialés anticipées, le marché visé par l'événement, la catégorie de visiteurs intéressés (jeunes, touristes spécialisếs, vacanciers, etc.), la ou les régions d'impact et l'importance de la promotion touristique aux niveaux régional, national et international, l'implication financière du secteur privé obtenue ou anticipée, les probabilités d'en arriver à un équilibre financier, l'importance de la visibilité de l'événement et sa couverture par les médias aux plans régional, national et international, les appuis reçus par les dirigeants locaux, les organisations, les hommes politiques ou autres et, aussi, la compétence et la crédibilité des concepteurs, des parrains de l'événement et des organisateurs.

La sollicitation en vue d'obterir une participation financière de l'entreprise privée se situe aussi à deux niveaux. Auptès des dirigeants que l'on retrouve au conseil d'administration et à la haute direction. Ces personnes bénéficient d'un pouvoir d'influence moins délicat à utiliser que celui des hommes politiques et sont en mesure d'approuver et d'orienter les décisions en fonction des objectifs stratégiques et politiques de l'organisation. Les autres intervenants, pouvant influencer la décision de seconder un événement, sont les professionnels de l'entreprise. Ceux-ci se retrouvent souvent à la direction du marketing ou des communications. Ils sont très concernés par les orientations de leur direction et les objectifs de vente et de visibilité de l'entreprise. Ils possèdent un pouvoir de décision plus important que leurs équivalents du secteur public et ont généralement plus d'affinité que ceux-ci avec les concepteurs et organisateurs d'événements en termes de promotion et de gestion.

Moins préoccupée par les paramètres d'évaluation des projets que par les objectifs qu'elle poursuit, l'entreprise privée décide de son implication dans la mesure où elle peut intégrer ceux-ci à sa stratégie de marketing, que son apport monétaire lui permet des retombées financières compa- rables à ses autres projets d'investissement: que le projet favorisera la visibilité de l'entreprise et des ses produits et qu'enfin. elle pourra éventuellement augmenter la part de son marché par l'impact de son implication auprès de la population touchée par le projet.

La définition d'objectifs et de normes précises d'evaluation a rendu plus complexe l'obtention de financement pour les événements sportifs ou culturels puisqu'elle nécessite des recherches auprès des gouvernements pour trouver le bon ministère et le bon programme, lorsque ce dernier existe. Elle requiert aussi la préparation de dossiers détaillés et documentés et les délais pour obtenir une décision sont importants. Parallèlement, il devient aussi de plus en plus difficile d'intéresser l'entreprise pri. vée compte tenu du très grand nombre de requêtes qu'elle reçoit et de l'orientation précise qu'elle se donne lors de ses interventions. Certains des concepteurs de projets et des vendeurs spécialisés de ceux-ci se sont donc efforcés de bien comprendre les préoccupations stratégiques des dirigeants des secteurs publics et privés. Ils ont aussi appris à bien comprendre les objectifs des programmes et les règles du jeu qui les régissent et, de ce fait, ils ont adapté le contenu du projet à être réalisé en fonc. tion du contenant exigé par les structures pouvant assurer son financement et sa survie, principalement durant sa phase de planification. De plus, des efforts considerables sont investis en vue de s'assurer le parrainage progressif d'individus ou d'organismes prestigieux ayant une influence sur les milieux des affaires ou politiques. Un tel engagement formel génère un intérêt allant en s'accroissant et permet éventuellement d'atteindre un niveau de développement du projet et d'implication auprès de la communauté nationale ou internationale qui ne permet pratiquement plus d'y renoncer, malgré les surprises que réserve la planification détaillée.

\section{Et la faisabilité dans tout ça?}

En principe, le processus d'approbation de projet passe successivement par des données de pré-faisabilité et de faisabilité qui sé voudront de plus en plus détaillées et tenteront d'analyser et d'évaluer avec le plus de précision possible les chances de réussite du projet à l'intérieur des paramètres de qualité, d'échéancier et de coút que ses conceptuers lui auront définis. Un second volet du travail devra aussi permettre d'évaluer l'impact de celui-ci sur son environnement êt sur les organismes touchés par sa réalisation. A la lumière des informations obtenues. des modifications devront sans doute être apportées aux objectifs et aux paramètres afin d'en arriver à définir un projet acceptable et accepté par l'ensemble des intervenants. Une fois cette étape achevée, le projet sera approuvé et sa pla. nification détaillée devrait normalement se situer à l'intérieur des limites de qualité, de coút et de temps lui ayant été allouées.

Malheureusement, la réalité de certains événements, et pas nécessairement les moins importants, a fait ressortir que les étapes prévues en phase de faisabilité ne donnaient pas toujours les résultats escomptés. Il y a plusieurs raisons pouvant expliquer ces échees ou demi-succès. Les particularités des projets à vocation culturelle ou sportive presentent un niveau de risque qui s'avère gếnéralement complexe à évaluer et à quantifier. La possibilité de se référer à des événements passés pour analyser la faisabilite de projets futurs n'est pas ce qu'il y a de plus facile, surtout lorsqu'il y a eu échec, puisque ces demiers sont rarement évalués a posteriori, que leurs dirigeants sont dispersés et que toute l'expertise écrite est difficilement retraçable. L'obligation des concepteurs de s'adapter, a priori, aux objectifs des bailleurs de fonds et de leur donner une image positive du projet les amène et c'est normal, à ne pas trop pousser la faisabilité technique des projets avant que des fonds soient assurés. Les intérêts des organismes publics ou privés de même que les objectifs et les normes d'approbation ne semblent pas accorder une priorité suffisante à la fajsabilité opérationnelle des projets. Les coûts des études de faisabilité par des tiers neutres spécialisés sont élevés et seul les gouvernements semblent être en mesure. encore une fois, de les assumer.

\section{En guise de conclusion}

Il n'est pas toujours avantageux d'adapter la théorie à la réalité. Cependant, il ne fait pas de doute qu'il y a place à amélioration dans la façon d'évaluer la faisabilité des projets à vocation socio-économique axés sur la culture et le sport. I] n'est pas acceptable que le financement de ceux-ci se fasse après coup sur le dos des contribuables par le biais des gouvernements ou sur celui des individus ou entreprises qui ont accepté de participer à sa réalisation. De mềme. l'importance des retombees positives à court et long termes et l'effet non négligeable d'une image négative pour une région. une province ou le pays méritent qué des efforts réels soient tentés pour améliorer le ratio de réussite de ces projets.

Il est certain que le succès de ces projets repose sur une collaboration réelle entre concepteurs, organisateurs et promoteurs privés et publics. Il apparaît donc souhajtable que les objectifs des programmes d'approbation de projets accordent une importance plus rigide à la faisabilité technique des projets. Un plus grand contact avec les opérationnels des secteurs public et privé favoriserait l'échange d'informations techniques et permettrait d'ếclairer les analystes de programmes recommandant l'approbation ou le rejet de ceux-ci. Des intervenants neutres devraient suivre le déroulement des étapes du projet et sensi-

(suite a la page 40) 


\section{RIO}

|suite de la page 111

ments et bijoux venus d'Afrique. En fait, la première "école" de samba, si associée de nos jours à l'idée du carnaval brésilien, ne naquit qu'en 1928 dans le quartier d'Estacio à Rio' d'autres, a Penha, à Mengueira, à Salgueiro et Leblon, suivirent bientôt.

A l'origine, une école de samba était constituée de résidents de chaque quartier ou de chaque colline entourant la ville. Leur suc= cès fut tel qu'elles se répandirent très rapidement par tout le pays. Par sa beauté et par la gaieté qu'il dégage, le défilé des écoles de samba constitue maintenant le point culminant du carnaval et du camaval de Rio en particulier, un spectacle incroyablement animé et coloré. Tous les ans, les ecoles élisent leurs chorégraphes et décident du theme de leurs prestations, basé sur un épisode de l'histoire du Brésil ou de son folklore.

Ainsi, chaque école présentera pendant le carnaval des chars allégoriques, des orchestres entiers armés de batteries et d'instruments de percussion ainsi que des centaines sinon des milliers de participants vẻtus, pour beaucoup, de riches costumes. De riches costumes que, de toute évidence, ils ne peuvent pas se payer. On évalue en effet que le coût moyen d'un costume d'une école de samba est de $80 \$$ US, soit le double du salaire minimum mensuel.

Ces coûts montent d'ailleurs en spirale et ne sont pas près de s'arrêter, en raison notamment de la concurrence effrénée que se livrent les diverses écoles pour s'approprier les prix que donne la minicipalité de Rio à la meilleure chanson de samba, aux plus beaux costumes, au plus beau char allégorique, à la meilleure performance générale. Un million de dollars US seraient ainsi dépensés ces derniêres années en de tels préparatifs. Certaines écoles renommées peuvent profiter du support financier de commanditaires alors que d'autres acceptent dans leurs rangs -contre rémunération- des outsiders, des résidents de quartiers plus riches et plus huppés; il demeure que beaucoup de personnes s'endettent lourdement en prévision des jours de carnaval et connaissent un réveil douloureux au matin du mereredi des Cendres...

\section{Un événement de plus en plus "tourné" vers l'extérieur}

Cette concurrence n'est pas sur le point de s'éteindre car elle fait dorénavant partie du produit. D'où l'intérêt de la municipalité de l'attiser. L'an dernier, quelques 40000 touristes, venus des Etats-Unis, d'Europe. d'Argentine, auraient afflué vers Rio pour connaitre les joies de la "grande fiesta". Avec la dévaluation de la monnale brésilienne, cela voulait dire d'importants achats de vêtements, de souliers. d'objets d'artisans et d'autres produits locaux: 80 millions de dollars. US auraient ainsi été injectés dans l'économie de Rio, seulement au cours des dix derniers jours du Carnaval!

Pour ne pas ëtre en reste, une quinzaine de grandes écoles ont formé une association indépendante d'ecoles de samba afin de mieux profiter des retombes financieres de l'événement et aussi des redevances des compagnies de disques qui publient chaque année -à raison de 700000 exemplaires!les plus populaires chansons de carnaval. Le gouvernement de Rio a lui-mème financé et construit un gigantesque Sambadrome de 90000 places (dessiné par Oscar Niemeyer, l'architecte de Brasilia) au coût de 15 millions de dollars. Mais les prix des billets y sont chers ( 455 par jour en moyenne), beaucoup plus que la très grande majorité de cariocas (habitants de Rio) ne peuvent payer. Et, comme les touristes ne peuvent tout de meme pas acheter tous les billets chaque jour et qu'il y a conséquemment un grand nombre d"invendus, on parle de scandale..

Quoi qu*il en soit, le carnaval de Rio illustre bien le cas d'une fête issue des forces "internes" d'une collectivité, de son imaginaire lé plus intime, qui est devenue un événement touristique, donc tourné vers l'extérieur, vers les attentes de populations externes, et les "bénéfices" "qu'elles apportent. Même si cela doit se faire au détriment des premiers et principaux artisans de la fềte.

\section{Niote}

(1) Dansison classique ouvrage, Voyage pittoresque et historique au Brésil. J.B. Debret a fait une excel. lente analyse et description de l'enfrudo.
HARMONIE

|suite de la page 25 |

biliser périodiquement les plus hautes instances aux difficultés opérationnelles de ceux-ci afin d'éclairer et d'accélérer les prisés de décision. Enfin, une centralisation des informations et des données relatives à ces événements spécifiques devrait être effectuée en vue d'en arriver à fournir des. références aux futurs concepteurs et promoteurs.

Il est certain que des coûts et des délais sont reliés à ces recommandations. Il faut payer un prix lorsque l'on vise la qualité. La réputation et la crédibilité d'une région, d'une province ou d'un pays ne peuvent s'établir que dans la mesure où les activités qui l'identifient sont de qualité. $f$

\section{Pdiférences}

Cet article a ete tecrit a partir du travall L'importance des atapes de faisabilité dans la ratisation de Quá. bee 1534-1984 et de projets similaires realisé par Gilles. Delorme dans le cadre du "Séminaire d'intégration $I^{\prime \prime}$ de le maitrise en pestion de projet-UOAM. Les documents consultes pour réaliser ce travail sont:

- Analyse sur la capacité du site au niveas humain, Qufbec, 1534-1984.

- LAVALIN, Circulation-Transport 1984-Plan de traneport et elrculation Qutbec, ote 1984, 23 févier 1984.

- Comité Cudbec 1534-1954, Rapport d'étude sur I'dvónement Qud́bac 1534-1984, Aoủt 1979

- Croissance de l'́buenement Qutbec 84. Commis sion rogionale Jean-Talon, Deuxieme trimestre 1994

- capon belanger onllaipe, gagnon comptables agrets. Etude de to gestion adminis: trative et financibre - Québec, Decembre 1984.

- ROCHE Ltée Groupe consiil. Etude des retembees exconomiques des dvenements publies du 450 e anniversaire de l'arrivere de Jacques Cartier au Canade - Rapport d'btape no 1, Dossier 3943-1000, Juin 1904 .

- ROCHE Ltée Groupe conseil, Etude des ratombebes diconomiques das ovónaments publics du 450 e anniversaire de l'arrivete de Jecques. $C$ ar tier au Canada. Dossier 3943-1000. Mars 1985.

- P.A. Cloutier, Gestion par projet, Automne 1985.

- Direction de l'evaluation - Agence canadienne de desweloppament intemational, Guide máthodologi. que a l'intention des equipes de projets qui ont a getrer des dvaluations. Oetobre 1980.

- Yves GRANDMONT M.Sc.A., Ing., La planification et le contrble en contexte de projet. Univétsité du Quebec aै Trois-Rivieres.

- Le projer Oubbec 15.341984 - Analyse du from. bre de visiteurs prévus ot des retombés éco nomiques escomptées, Office du tourisme du Canada.

- Rapport du Comité sur la responsabilité sociale de l'entreprist. Chambre de commerce de Canada, Novembre 1982 